\title{
Caracterização das pesquisas sobre a próxima rede global de comunicação: a Internet Quântica
}

\author{
Moniele S. Sousa ${ }^{1}$, Alex F. de Paulo ${ }^{2}$, Flávio de O. Silva ${ }^{1}$, João H. de S. Pereira ${ }^{1}$ \\ ${ }^{1}$ Programa de Pós-graduação em Ciência da Computação \\ Universidade Federal de Uberlândia (UFU) \\ 38408-100 - Uberlândia - MG - Brazil \\ ${ }^{2}$ Faculdade de Informação e Comunicação \\ Universidade Federal de Goiás, \\ Goiânia, GO - Brazil
}

\begin{abstract}
The Quantum Internet, a network that connects quantum devices between any two points on Earth, has been transforming the scenario of today's Internet. In this research, we propose an approach to characterize the evolution of this knowledge, having a database of scientific publications between the years 1984 and 2020, on the Scopus and Web Of Science platforms. Our results showed that studies have a clear predominance of publications in journals related to physics, with China and the EUA. Based on the analyzed scientific bases, the results also show that research is still being researched in embryonic stages, having a long way to be explored.
\end{abstract}

Resumo. A Internet Quântica, uma rede que conecta dispositivos quânticos entre quaisquer dois pontos da Terra, vem transformando o cenário da atual internet. Nesta pesquisa, propomos uma abordagem de caracterização da evolução desse conhecimento, tendo uma base de publicações científicas entre os anos de 1984 e 2020, nas plataformas Scopus e Web Of Science. Nossos resultados demonstraram que os estudos tem predominância nítida de publicações em periódicos relacionados à física, sendo dominante desse conhecimento, China e EUA. Baseado nas bases científicas analisadas, os resultados também mostram que as pesquisas ainda se encontram em estágios embrionários, tendo um longo caminho a ser explorado.

\section{Introdução}

A Internet Quântica tem causado um efeito revolucionário no mundo quântico, abrindo relevantes novos recursos de comunicação e computação. A Computação Quântica tem papel fundamental nessa expansão devido anteceder os estudos sobre Internet Quântica. Esse movimento surgiu, proposto nos anos 80, demonstrando seu potencial para solucionar problemas que antes não eram possíveis por meio de computadores clássicos [Feynman 1982]. Desde então, a Computação Quântica tem feito emergir pesquisas que viabilizem essa interligação via Internet Quântica [Caleffi et al. 2018].

Em busca dessa maior eficiência de processamento, a parte computacional teve um grande impulso. Em novembro de 2017, foi construído um processador de 50 qubits e testado com sucesso [Castelvecchi 2017]. Em seguida, o Google lançou um processador de 72 qubits e em 2019, um processador nomeado Sycamore de 53 qubits levou seus 
criadores a requisitarem o marco de alcançar a supremacia quântica, pela primeira vez na humanidade [Arute et al. 2019].

Estudos realizados pelos pesquisadores de Massachusetts Institute of Technology - MIT e da Northwestern University - NU concentraram seus esforços em como teletransportar qubit de longa distância com alta fidelidade a partir de Computadores Quânticos [Lloyd et al. 2004]. A União Européia, em abril de 2017, investiu 1 bilhão de euros e empenhou seus esforços nas pesquisas sobre Tecnologias Quânticas [Gibney 2017]. Em junho do mesmo ano, a China realizou um teste de comunicação de $1200 \mathrm{~km}$ entre o satélite Micius e estações terrestres [Liao et al. 2017], [Yin et al. 2017]. Esses estudos apontam uma perspectiva de comunicaçãoo quântica entre pontos distantes da Terra.

Baseado na lacuna teórica, não se dispõe na literatura estudos que caracterizasse essa evolução da comunicação de Internet Quântica. Neste momento, não é tarefa fácil caracterizar essa futura tecnologia, e prever quais os seus mais variados impactos. Assim, esse artigo propõe criar uma base de conhecimentos científicos, caracterizando a próxima rede global de comunicação, mapeando sua evolução ao longo da história, a partir de uma análise das estatísticas descritivas. Entender esta caracterização e evolução histórica torna-se importante e necessário devido os promissores impactos do uso futuro desta tecnologia em diversos setores da sociedade, por exemplo, e não limitando a, saúde, comunicação e segurança. A tamanha dimensão destes impactos tem potencial de remodelar até mesmo os caminhos de pesquisas e ensino na área em computação, redes de computadores, sistemas distribuídos e segurança cibernética, dentre outros.

\section{Trabalhos Relacionados}

Conforme os avanços da tecnologia quântica amadurecem, tem-se atraído com sucesso gigantes da tecnologia, como IBM, Google e Intel, a participar da chamada corrida quântica. Um marco muito significativo foi alcançado no final de 2019 por um grupo de pesquisadores que anunciou a supremacia quântica, em um evento importante que possibilitou um processador quântico realizar uma tarefa computacional que estava além da capacidade de qualquer computador clássico [Debenedictis 2020]. E recentemente, em um laboratório, superou-se o alcance de $1,3 \mathrm{Km}$ e alcançou-se o emaranhamento de dois conjuntos atômicos por meio da transmissão de fótons através de fibras ópticas no raio de 22 e $50 \mathrm{Km}$ [Llewellyn et al. 2020], [Yu et al. 2020].

Uma versão do roteiro detalhando as etapas da primeira rede quântica genuína prevista para 2020 já foi desenvolvido pela equipe de pesquisadores da Universidade de Delft [Wehner et al. 2018], que ligará quatro cidades na Holanda, com esforços do projeto Quantum Internet Alliance.

A Internet Quântica traz como princípio uma gama de possibilidades que não era possível na comunicação clássica, remodelando-a, de tal forma que, com a Internet Quântica, é possível complementar a Internet atual ou definitivamente utilizar somente meios quânticos [Castelvecchi 2018]. Apoiado nessa nova comunicação, pesquisadores abrem um universo de vantagens que tem como objetivo suprir as carências que a Internet convencional possui, uma delas é a segurança das informações trafegadas. Uma das principais aplicações conhecidas da Internet Quântica é a distribuição de chave quântica $(Q K D)$, onde duas partes produzem uma chave única e aleatória, que não é conhecida por um terceiro [Wehner et al. 2018]. 


\section{Metodologia}

A presente pesquisa é de natureza descritiva e utiliza estatística para análise sobre domínio do conhecimento científico. Quanto à natureza esta pesquisa se enquadra como sendo quantitativa devido ao uso de base de dados de artigos científicos, esses que são fontes fidedignas e relevantes, e que permite análises estatísticas descritivas para viabilizar uma análise exploratória dos dados coletados sobre o movimento da Internet Quântica.

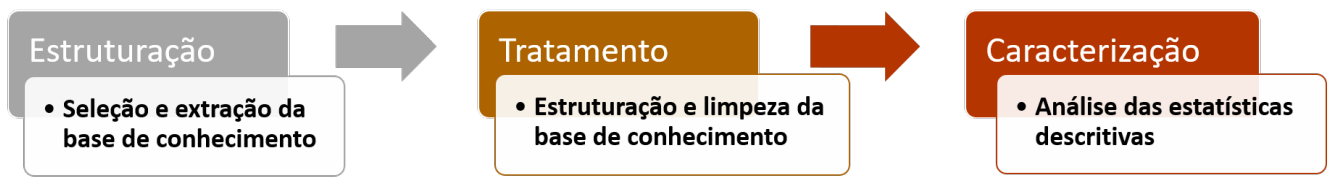

Figura 1. Etapas metodológicas

Temos na Figura 1, a primeira etapa, que utilizou como fontes de coleta de dados, as publicações na base de dados Scopus e Web Of Science. Ambas ferramentas de pesquisa foram escolhidas em razão de sua confiabilidade científica e fornecer acesso baseado em assinatura a vários bancos de dados que oferece dados confiáveis e abrangentes de citações em diversas áreas do conhecimento.

As coletas se deram a partir de todas publicações existentes (Article or Conference Paper), dentre 1984 até o ano de 2020 em cada plataforma, para os termos "quantum internet" e "quantum network". A primeira coleta de dados foi realizada na Scopus, com análise de 2008 artigos de publicados em um único arquivo. Já a segunda coleta realizada na base Web Of Science, totalizou 1011 publicações em três arquivos de até 500 publicações, limitação essa da base. Na segunda etapa, com os arquivos coletados, iniciou-se o processo de importação de cada arquivo e a interseção de ambos, reunindo todas as informações necessárias.

Foi possível identificar um total de 3019 publicações entre artigos de revistas e proceedings. Ainda na segunda fase, um processo de limpeza dos dados foi fundamental, em razão dos dados terem registros em duplicidade, ao passo que foram eliminadas para evitar uma análise incorreta, no total 815 publicações. Por fim, após esses primeiros recortes, a amostra tratada para a análise descritiva tem um total de 2204 registros. Os autores, palavras chaves e afiliações citados estavam dispostos cada um dentro de uma mesma célula, então foi necessário separá-los em células diferentes para fazer o cruzamento de dados.

A fase final, a caracterização da Internet Quântica, baseou-se na análise das estatísticas descritivas gerada pelos arquivos tratados. Isso permitiu explorar e aplicar várias técnicas para descrever e sumarizar melhor os dados por meio de análise de frequência, gráficos temporais, histogramas, ranqueamento de variáveis e atributos e etc.

\section{Análise de Resultados}

Buscando entender o contexto geral do estado da arte sobre Internet Quântica, os dados permitiram gerar visualizações para análises descritivas dos dados selecionados.

As publicações até 2008 se mantiveram em um patamar médio de 11 artigos por ano. Nos anos de 1985 até 1988, 1992 e 1994 não se teve publicações, sendo que somente 
a partir de 1995 é possível visualizar uma constância, com origem na Universidade de Ciência e Tecnologia da China, e Academia Austrıca de Ciências. Chamamos essa etapa de Fase A (Figura 2).

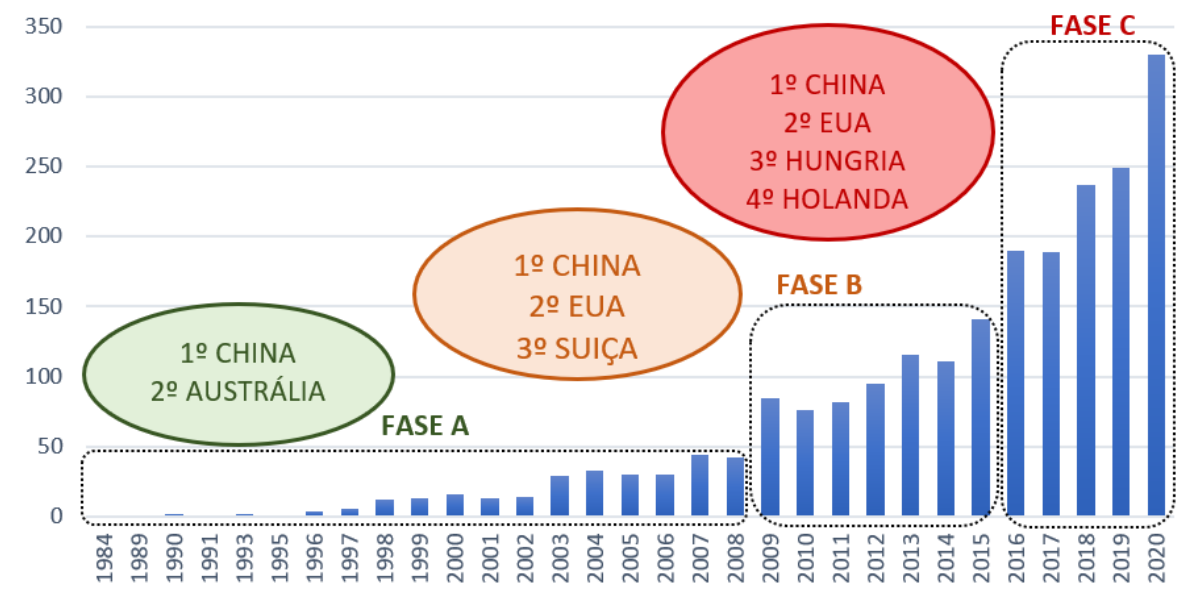

Figura 2. Volumetria de publicações de Internet Quântica

Na Fase B, entre 2009 e 2015, há um crescimento considerável de publicações na área, em média 100 por ano, na Universidade de Ciência e Tecnologia da China, na Academia Austriaca de Ciência, na Universidade de Harvard no EUA, e na Universidade de Genebra. Por último, a Fase C, entre 2016 e 2020, o crescimento de trabalhos acerca desse tema cresceu exponencialmente, tendo 2020 o maior volume com 300 publicações e uma média de 239 por ano, ressaltando-se a Universidade de Ciência e Tecnologia da China, Universidade de Harvard nos EUA, Universidade de Tecnologia e Economia de Budapeste, e Universidade de Tecnologia de Delft. Foi notório a análise ao longo dos anos que o tipo de conhecimento pesquisado tem raízes na física quântica, sendo mais de $70 \%$ das publicações originadas nesta área. Destacando-se os países como China, Austrália, EUA, Suiça, Hungria e Holanda que predominam esse conhecimento.

Na Figura $3 b$ tem-se a evolução de publicações por autor. É notável uma oscilação ao longo da história, ficando frequente a partir de 2014. Já na figura 3a é analisado os autores que mais se somaram em quantidade de publicações desde 1984 até 2020. O autor que se destaca é o físico chinês, Guo Guangcan. Professor da Universidade de Ciência e Tecnologia da China (USTC) e da Universidade de Pequim (PKU). Ele é um acadêmico que no total publicou 45 artigos entre 1984 e 2020. Em segundo lugar, com 35 publicações, Pan Jianwei, um físico quântico chinês renomado por sua dedicação no tema de entrelaçamento quântico. Ele faz parte da Academia Chinesa de Ciências e da Academia Mundial de Ciências, atuando como vice-presidente da (USTC). Com 30 publicações, Mikhail Lukin é um físico teórico e experimental russo e professor da Universidade de Harvard.

A principal revista de física do mundo é foco nas publicações dessa pesquisa (Figura 4). Physical Review Letters (PRL) desde 1958, tem contribuído para avançar e difundir o conhecimento da física, publicando pesquisas ilustres em todos os campos da física. Na Figura 4 temos a PRL totalizando 32 publicações. Em seguida, a Physical Review é uma das mais antigas e mais respeitadas revistas científicas que publica pesquisas em todos os aspectos da física. Inclusive nas áreas de rápida evolução da física atômica, 


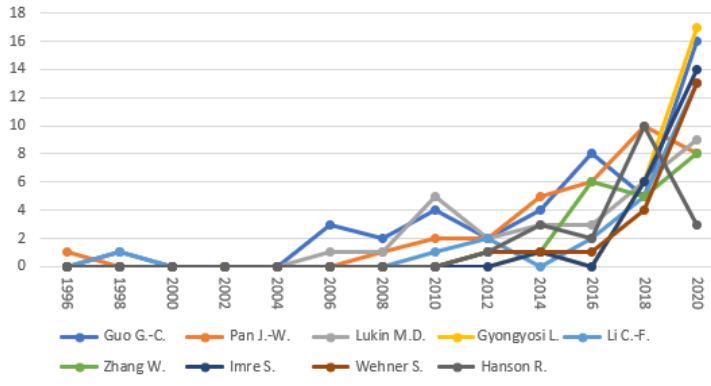

(a) Histórico de publicações por autor

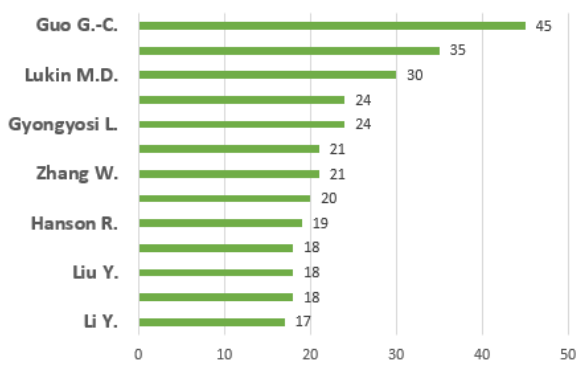

(b) Autor com maior número de publicações na história

Figura 3. Evolução dos Autores

molecular, e óptica e informações quânticas. A predominância nítida de publicações em periódicos relacionados à física, induz ao entendimento que os estudos sobre Internet Quântica ainda se encontram em estágios embrionários, e que ainda tem um longo caminho a percorrer até sua implementação e consolidação em outras áreas.

Figura 4. Distribuição das publicações entre as principais revistas

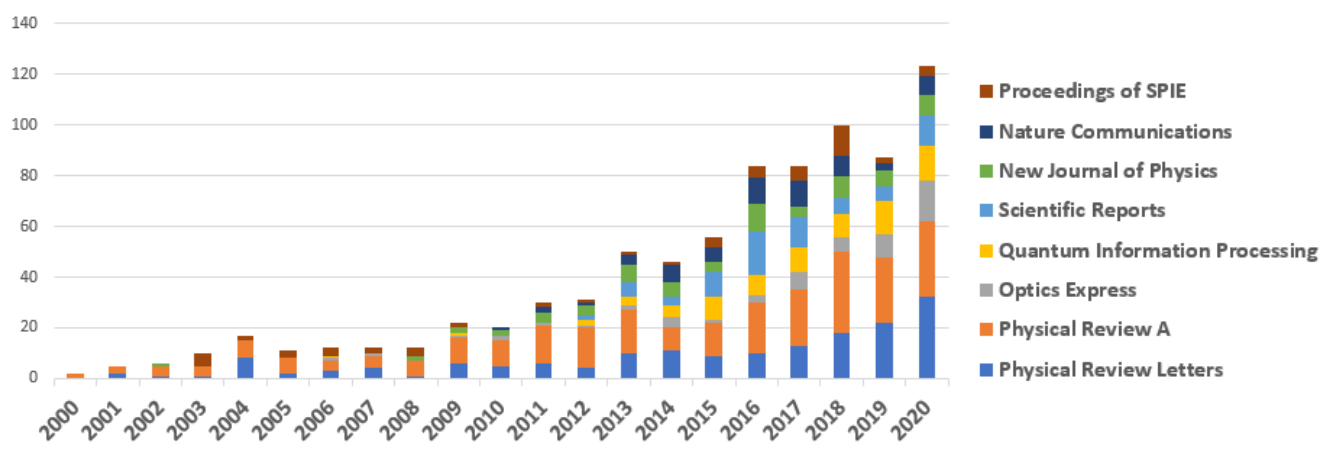

\section{Conclusão}

Neste trabalho, foi proposta uma abordagem de análise descritiva da Internet Quântica. O objetivo principal dessa abordagem é caracterizar o tema mapeando sua evolução ao longo da história.

Como conclusões preliminares após analisar os resultados apresentados na Seção 4, tem-se que a Internet Quântica possui, atualmente, mais de 70\% das publicações originadas pela área da fisica, sendo ainda um campo de conhecimento em estágio embrionário para outras áreas. Com foco de pesquisadores ao redor do mundo, a Universidade de Ciência e Tecnologia da China, a Universidade de Harvard nos EUA, a Universidade de Tecnologia e Economia de Budapeste, e a Universidade de Tecnologia de Delft lideram o conhecimento e investigação sobre o tema conforme vimos nos resultados apresentados.

A análise das bases de conhecimento exploradas, nesta pesquisa, induz ao entendimento que a Internet Quântica provocará enormes disrupções, na próxima década, em Ciência e Tecnologia (C\&T) e Sociedade. Por exemplo, poderá afetar, de forma significativa, o sistema de ensino e comunidade acadêmica em diversas áreas de tecnologia. Não foi objetivo desta pesquisa explorar a dimensão destes impactos, sendo este entendimento proposto para investigações em trabalhos futuros. 
Adicionalmente, como perspectivas de trabalhos futuros, sugere-se o complemento de uma análise quantitativa, baseado em rotas de conhecimento tecnológico, e uma análise qualitativa, baseada em entrevistas com especialistas da área, a fim de alcançar maiores detalhes da evolução da Internet Quântica e seus respectivos impactos.

\section{Referências}

Arute, F., Arya, K., Babbush, R., Bacon, D., Bardin, J. C., Barends, R., Biswas, R., Boixo, S., Brandao, F. G., Buell, D. A., et al. (2019). Quantum supremacy using a programmable superconducting processor. In Nature, pages 505-510. Nature Publishing Group.

Caleffi, M., Cacciapuoti, A. S., and Bianchi, G. (2018). Quantum internet: From communication to distributed computing! In Proceedings of the 5th ACM International Conference on Nanoscale Computing and Communication, page 4. Association for Computing Machinery.

Castelvecchi, D. (2017). Ibm's quantum cloud computer goes commercial. In Nature, page 159.

Castelvecchi, D. (2018). The quantum internet has arrived (and it hasn't). In Nature, pages 289-292. Nature Publishing Group.

Debenedictis, E. P. (2020). Beyond quantum supremacy. In Computer, pages 91-94. IEEE Computer Society.

Feynman, R. P. (1982). Simulating physics with computers. In International Journal of Theoretical Physics, pages 133-153.

Gibney, E. (2017). Europe's billion-euro quantum project takes shape. In Nature, page 16.

Liao, S.-K., Cai, W.-Q., Liu, W.-Y., Zhang, L., Li, Y., Ren, J.-G., Yin, J., Shen, Q., Cao, Y., Li, Z.-P., et al. (2017). Satellite-to-ground quantum key distribution. In Nature, pages 43-47. Nature Publishing Group.

Llewellyn, D., Ding, Y., Faruque, I. I., Paesani, S., Bacco, D., Santagati, R., Qian, Y.J., Li, Y., Xiao, Y.-F., Huber, M., et al. (2020). Chip-to-chip quantum teleportation and multi-photon entanglement in silicon. In Nature Physics, pages 148-153. Nature Publishing Group.

Lloyd, S., Shapiro, J. H., Wong, F. N. C., Kumar, P., Shahriar, S. M., and Yuen, H. P. (2004). Infrastructure for the quantum internet. In SIGCOMM Comput. Commun. Rev., pages 9-20.

Wehner, S., Elkouss, D., and Hanson, R. (2018). Quantum internet: A vision for the road ahead. In Science. American Association for the Advancement of Science.

Yin, J., Cao, Y., Li, Y.-H., Liao, S.-K., Zhang, L., Ren, J.-G., Cai, W.-Q., Liu, W.-Y., Li, B., Dai, H., et al. (2017). Satellite-based entanglement distribution over 1200 kilometers. In Science, pages 1140-1144. American Association for the Advancement of Science.

Yu, Y., Ma, F., Luo, X.-Y., Jing, B., Sun, P.-F., Fang, R.-Z., Yang, C.-W., Liu, H., Zheng, M.-Y., Xie, X.-P., et al. (2020). Entanglement of two quantum memories via fibres over dozens of kilometres. In Nature, pages 240-245. Nature Publishing Group. 